\title{
Un nouvel essor pour le dialogue mécanique-physique
}

Des actions nouvelles en France se développent à l'interface physique - mécanique. Avant de les évoquer en seconde partie, rappelons plusieurs phases du dialogue entre mécanique et physique.
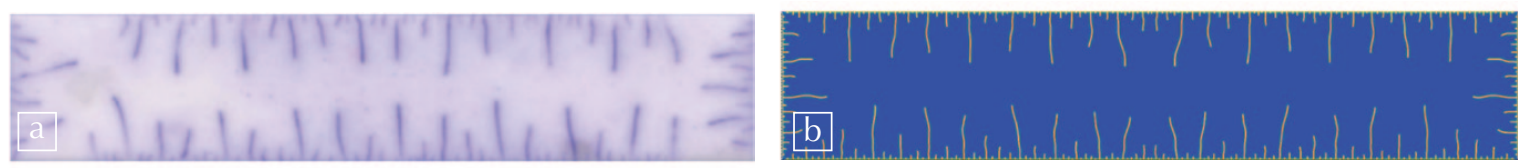

1. Réseau de fissures induites par un choc thermique d'une plaque fragile.

a) Résultats expérimentaux obtenus après trempe thermique d'une plaquette en céramique (C. Jiang et al., Acta Materiala 60 (2012) 4540 ).

b) Résultats numériques obtenus par solution d'un modèle d'endommagement à gradient, version régularisée du modèle de rupture de Griffith

(P. Sicsic, B. Bourdin, J.J. Marigo et C. Maurini, soumis à Phys. Rev. Lett.). La forme des fissures est déterminée par la minimisation de la somme de l'énergie élastique et de l'énergie de surface. Leur nucléation peut s'expliquer comme un phénomène de bifurcation à partir du matériau sain.

\section{Références}

- Voir aussi Reflets de la physique 17 (2009) $32-33$.

2•É. Guyon et D. Bideau, Reflets de la physique 12 (2008) 5-7.

3• D. Bideau, É. Guyon et J. Pullino, « Le tas de sable : du désordre qui rassemble ", La revue pour l'histoire du CNRS, 22 (2008), http://histoire-cnrs. revues.org/ 8272

4 Des archives relativement complètes de la vie de cette communauté sont gérées au laboratoire IUSTI du CNRS

à Marseille (contact : jeanne.pullino @polytech.univ-mrs.fr).
Quelques grands noms évoquent la fécondité d'un intérêt commun : Joseph Louis Lagrange (1736-1813), Pierre Simon Laplace (1749-1827) et Thomas Young (1773-1829), autour de la physique des surfaces. James Clerk Maxwell (1831-1879) pose les bases de la photoélasticité... et un de ses premiers articles porte sur la chute libre d'une feuille ! Lord Kelvin (1824-1907) étudie la thermodynamique de l'élasticité. Heinrich Hertz (1857-1894), antérieurement à ses apports à l'électromagnétisme, fait une thèse reconnue sur la déformation élastique au contact entre deux sphères. Un peu plus tard, Albert Einstein calcule la viscosité d'une suspension de particules solides, et ne dédaignera pas de s'intéresser à la distribution de feuilles dans le fond d'une tasse de thé mise en rotation. Werner Heisenberg portera un intérêt constant à la turbulence, dont l'étude était son sujet de thèse. La thèse de Max Born portait, elle, sur la confirmation expérimentale des équations des déformations de poutres élastiques (l'Elastica d'Euler).

$\mathrm{Au}$ cours du $\mathrm{XX}^{\mathrm{e}}$ siècle, les grands problèmes de la "physique moderne " conduisent les physiciens occidentaux, accaparés en particulier par la physique nucléaire et des particules, à négliger souvent les systèmes dynamiques et la mécanique des milieux déformables. On observe alors une séparation qui se fait de plus en plus nette entre mécanique et physique.

À partir des années 70, un intérêt renouvelé des physiciens les ramène vers des questions qui sont du domaine de la mécanique des milieux continus, mais presque exclusivement en mécanique des fluides (où les physiciens parleront plus volontiers d' « hydrodynamique physique »). L'étude de la matière offre des ouvertures nouvelles (hélium superfluide, cristaux liquides, polymères, matériaux granulaires). La physique non linéaire permet des approches théoriques nouvelles à des problèmes tels que la convection de RayleighBénard. Les outils physiques tels que le laser conduisent à des investigations multi-échelles résolues temporellement de la dynamique des fluides et des solides. Un nouveau souffle vient aussi de la biophysique, où les notions de mécanique prennent une place croissante.

Si la frontière entre mécaniciens et physiciens des fluides s'est considérablement estompée, c'est encore insuffisamment le cas en ce qui concerne la mécanique du solide, si l'on excepte la métallurgie physique qui s'était développée dans l'après-guerre. Ceci justifie les actions communes mises en place par les deux communautés.

Cette interface entre mécanique et physique est actuellement dynamisée au niveau de la Société Française de Physique par l'organisation de rencontres thématiques communes avec l'AFM (Association Française de Mécanique) depuis 2005, avec des journées d'études qui sont très suivies. On notera aussi un minicolloque sur la mécanique des objets élancés [1] aux Journées de la Matière Condensée en 2012, et la réciproque : un minicolloque "rencontres mécanique-physique " au Congrès français de mécanique d'août 2013.

Ces actions sont suivies et encouragées grâce au GDR commun MéPhy (Mécanique et physique des systèmes complexes, www.pmmh.espci.fr/ mephy), recréé cette année en tant que structure mixte interinstituts du CNRS, soutenu conjointement par l'INSIS et l'INP. Mais d'autres actions telles que les MIAMs (milieux aléatoires macroscopiques [2-4]), qui s'étaient mises en place en 1980 et accueillent des GDR tels que $\mathrm{MeGe}$ (mécanique géo-expérimentale) ou Transnat (Transport solide naturel), s'efforcent de vivre 

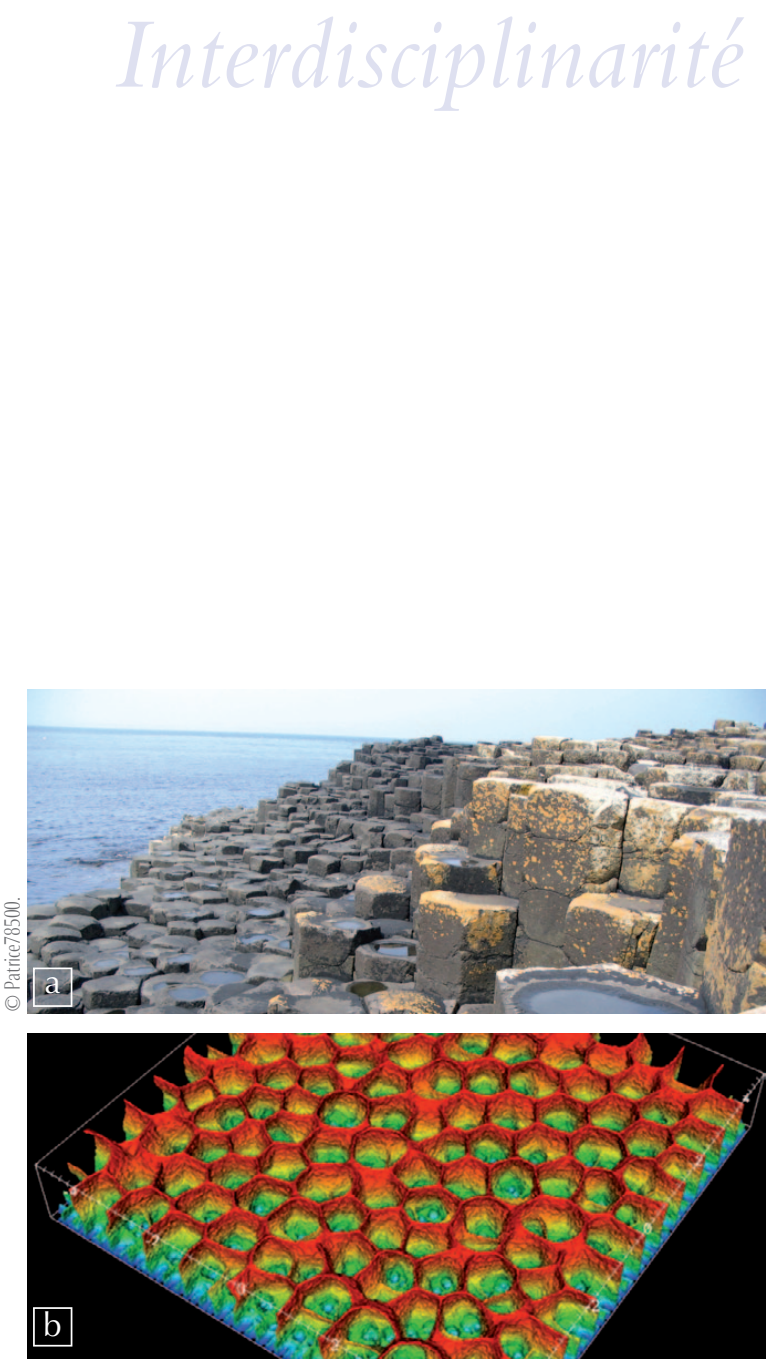

2. Réseau de fissures induites par un choc thermique d'un matériau tridimensionnel.

a) La Chaussée des géants, en Irlande du Nord, constituée de la juxtaposition de prismes de lave refroidie. La fracturation hexagonale en colonnes résulte de la contraction thermique rapide d'une coulée de lave basaltique fluide, expulsée à l'ère tertiaire.

b) Simulation numérique : cas tridimensionnel d'un choc thermique, avec la formation de structures hexagonales. Ce type de réseau de fissures est observé à des échelles très différentes : revêtements thermiques en céramique des turbines, formations géologiques (colonnes basaltiques, voir (a)), boue séchée. lci, le réseau de fissures hexagonales est créé par choc thermique d'un matériau fragile. Les résultats sont obtenus par simulation numérique directe par une méthode énergétique et discrétisation aux éléments finis, sans aucune hypothèse sur la géométrie des fissures. Ils montrent que le réseau final est le résultat du mûrissement d'un motif d'amorce de petite longueur d'onde (la couleur représente la distance de la surface d'application du choc).

un dialogue auquel souscrivent les scientifiques des deux communautés autour de problèmes communs, voire avec des approches qui s'enrichissent les unes les autres.

Les deux exemples présentés dans les figures 1 et 2 montrent comment la simulation numérique arrive maintenant à bien rendre compte de phénomènes de fissuration. I

Benoît Roman (benoit.roman@espci.fr) PMMH, ESPCI, 10 rue Vauquelin, 75231 Paris Cedex 05 